\title{
Technologiebewertung digitaler Gesundheitsanwendungen für Refundierungsentscheidungen
}

\author{
Reinhard JeindI (D) - Claudia Wild
}

Eingegangen: 12. April 2021 / Angenommen: 5. August 2021

(C) Der/die Autor(en) 2021

\section{Zusammenfassung}

Hintergrund Für die meisten digitalen Gesundheitsanwendungen (DiGA) liegt wenig Evidenz zum Nutzen vor. Bisher verfügbare Bewertungsinstrumente umfassen häufig nicht alle Domänen eines vollen Health Technology Assessments (HTA). Die Evaluation von DiGA erfordert zusätzlich technologiespezifische Aspekte. Ziel dieser Arbeit war es, verfügbare Bewertungsinstrumente zu analysieren und daraus einen Bewertungsprozess zu konzipieren.

Methodik Durch eine systematische Literatursuche wurden 6 Bewertungsinstrumente für DiGA ausgewählt und analysiert. Es wurde eine Handsuche zur Beschreibung der Strategien einzelner Länder im Umgang mit DiGA durchgeführt.

Ergebnisse Studiendesigns wurden in 4 der analysierten Bewertungsinstrumente beschrieben. Eine Risikoklassifikation wurde in 1 Bewertungsinstrument vorgeschlagen. Aspekte der künstlichen Intelligenz wurden in 1 Bewertungsinstrument erhoben. Einzelne Länder weisen unterschiedliche Strategien zur Refundierung von DiGA auf.

Schlussfolgerungen Die Bewertungsinstrumente für DiGA zeigen eine große Heterogenität. Rezente Entwicklungen verschiedener Länder zeigen Bestrebungen, Regelungen auf nationaler Ebene zu finden. Für einen Bewertungsprozess von DiGA empfiehlt sich ein abgestuftes Vorgehen unter Berücksichtigung von Risikoklassen mit anschließender Bewertung relevanter HTA-Aspekte.

R. Jeindl $(\bowtie) \cdot C$. Wild

Austrian Institute for Health Technology Assessment GmbH

(AIHTA), Garnisongasse 7/20, 1090 Wien, Österreich

reinhard.jeindl@aihta.at
Schlüsselwörter Digitale Gesundheitsanwendungen . Assessment Frameworks · Refundierung · mHealth · Digitale Gesundheit

Technology assessment of digital health
applications for reimbursement decisions

\section{Summary}

Background For most digital health applications (DiGA) only limited evidence of benefit is available. Currently available assessment frameworks do not cover all domains of a full health technology assessment (HTA). Additionally, technology-specific aspects are required for the evaluation of DiGA. This work aimed to analyze the available assessment frameworks and design an evaluation process for DiGA.

Methods By a systematic literature search six assessment frameworks for DiGA were selected and analyzed. A hand search for strategies on DiGA of selected countries was conducted.

Results Of the analyzed assessment frameworks four described study designs. One assessment framework proposed a risk classification of DiGA. Aspects of artificial intelligence were assessed by one assessment framework. The analyzed countries have differing strategies for reimbursement of DiGA.

Conclusion Assessment frameworks for DiGA are very heterogeneous. There are efforts to find regulations for DiGA on a national level. When evaluating DiGA, a staged approach considering risk classes with subsequent evaluation of relevant HTA aspects is recommended.

Keywords Digital Health Applications - Assessment Frameworks · Reimbursement $\cdot$ MHealth $\cdot$ Digital Health 


\section{Einleitung}

Digitale Technologien sind Teil unseres Alltags. Dabei hat in den letzten Jahren insbesondere die Nutzung mobiler digitaler Technologien an Bedeutung gewonnen und macht inzwischen den Großteil der Nutzung digitaler Technologien aus [1]. Diese vermehrte Nutzung digitaler Technologien betrifft auch den Gesundheitsbereich. Das Angebot reicht von rein informativen Anwendungen über Anwendungen mit diagnostischen Funktionen sowie Anwendungen für therapeutische Zwecke.

Mit dem Einsatz von digitalen Gesundheitsanwendungen (DiGA) sind große Erwartungen verbunden: DiGA haben das Potenzial, das Monitoring und das Management von Krankheiten zu verbessern, und könnten unnötige Wege für Patient*innen und Therapeut*innen vermeiden. Durch eine Fernüberwachung, insbesondere bei chronischen Erkrankungen, könnten Krankenhausaufenthalte vermieden werden. Bei kontinuierlich gemessenen Vitalparametern könnte eine Entgleisung eines Werts rasch erkannt und entsprechende Maßnahmen könnten gesetzt werden. Für die Mehrzahl der verfügbaren DiGA liegt bislang aber wenig Evidenz zum tatsächlichen Nutzen vor. Die gründliche Evaluation einer DiGA erfordert zusätzlich zu einer Nutzen-Risiko-Abwägung auch technologiespezifische Aspekte. Bisher verfügbare Bewertungsinstrumente umfassen häufig nicht alle Domänen eines vollen Health Technology Assessments (HTA). Entscheidungsträger*innen werden somit vor neue Herausforderungen in der Evaluation dieser Applikationen gestellt.

Durch die neue EU-Verordnung 2017/745 über Medizinprodukte, die im Mai 2021 in Kraft tritt, ist auch medizinische Software als Medizinprodukt einzustufen, wenn die Software laut Hersteller dazu bestimmt ist, medizinische Zwecke zu erfüllen [2]. Zusätzlich zu Medizinproduktsoftware („medical device software“, etwa die Software eines EKG-Gerätes) kann auch Software als Medizinprodukt („software as a medical device", etwa eine DiGA mit diagnostischen und/ oder therapeutischen Funktionen) betrachtet werden. Durch diese neue EU-Verordnung 2017/745 wird es zu einer Höherstufung der Risikoklasse vieler digitaler Gesundheitsanwendungen kommen [3].

Erste DiGA werden bereits als medizinische Leistung von einzelnen Krankenkassen in Deutschland, den Niederlanden und Belgien in Selektivverträgen übernommen. Für eine verbesserte Regulierung von medizinischer Software wird eine generelle, nationale Refundierung von geeigneten DiGA empfohlen [4, 5]. In Europa hat man in einzelnen Ländern begonnen, einen Prozess der Registrierung aufzusetzen, der infolge für einzelne DiGA auch zu einer Refundierung durch Sozial- und Krankenversicherungen führen kann.

Die Intention dieser Arbeit, die auf einem ausführlichen Projektbericht basiert [6], war es, eine kritische
Analyse verschiedener Bewertungsinstrumente für DiGA durchzuführen und eine Übersicht unterschiedlicher Zugänge einzelner europäischer Länder bei der Einführung und Implementierung von DiGA im Gesundheitswesen aufzuzeigen. Daraus abgeleitet, verfolgte die Arbeit das Ziel, eine Orientierungshilfe über den Umgang mit DiGA für Entscheidungsträger*innen zu erstellen.

\section{Methodik}

Suche nach Bewertungsinstrumenten: In einem ersten Schritt wurde eine systematische Literatursuche (im Juni 2020) in den Datenbanken Medline, Embase, Cochrane und der HTA Datenbank (Centre for Reviews and Dissemination) nach Bewertungsinstrumenten für DiGA durchgeführt und durch eine zusätzliche Handsuche ergänzt. Die systematische Suche diente einer Aktualisierung des systematischen Reviews von Vis et al. [7] zu Bewertungsinstrumenten für DiGA. Bewertungsmodelle von DiGA, die in englischer oder deutscher Sprache ab März 2018 publiziert wurden, wurden inkludiert.

Um weitere Bewertungsinstrumente für DiGA zu identifizieren, wurde ebenfalls im Juni 2020 eine Anfrage an alle Mitglieder des International Network of Agencies for Health Technology Assessment (INAHTA) gestellt. Dabei wurde erhoben, welche Bewertungsinstrumente von verschiedenen HTA-Institutionen als adäquat für DiGA erachtet werden und ob die jeweilige HTA-Institution an einer Entwicklung oder Adaptierung eines Bewertungsinstruments für DiGA arbeitet.

Auswahl der Bewertungsinstrumente und Datenextraktion: Aus der systematischen Suche und den Antworten der INAHTA Befragung wurden jene Bewertungsinstrumente für eine genauere Analyse ausgewählt, die aktuell (ab März 2018 publiziert), ausgereift (fortgeschritten in der Entwicklung), und für welche eine Anwendbarkeit (an konkreten DiGA) zu erwarten war. Bereits etablierte Bewertungsinstrumente wurden nicht gewählt, wenn diese zwar für den Bereich eHealth aufgestellt sind, jedoch die Entwicklungen im Bereich mobiler DiGA („mHealth“) nicht ausreichend berücksichtigten.

Die ausgewählten Bewertungsinstrumente wurden miteinander verglichen und auf Ähnlichkeiten und Unterschiede analysiert. Kriterien für die Datenextraktion aus den Bewertungsinstrumenten waren Institution/Land, Finanzierung, Zeitpunkt der Veröffentlichung, Zielgruppe, Entwicklungsstatus, Anwendungsbereich, Berücksichtigung von Risikoklassen, Evidenzerfordernisse, Zeitpunkt der Bewertung, Format, Bewertungsdomänen, technologiespezifische Aspekte, Involvierung Patient*innenvertretung und sonstige Spezifika des Bewertungsinstruments. 


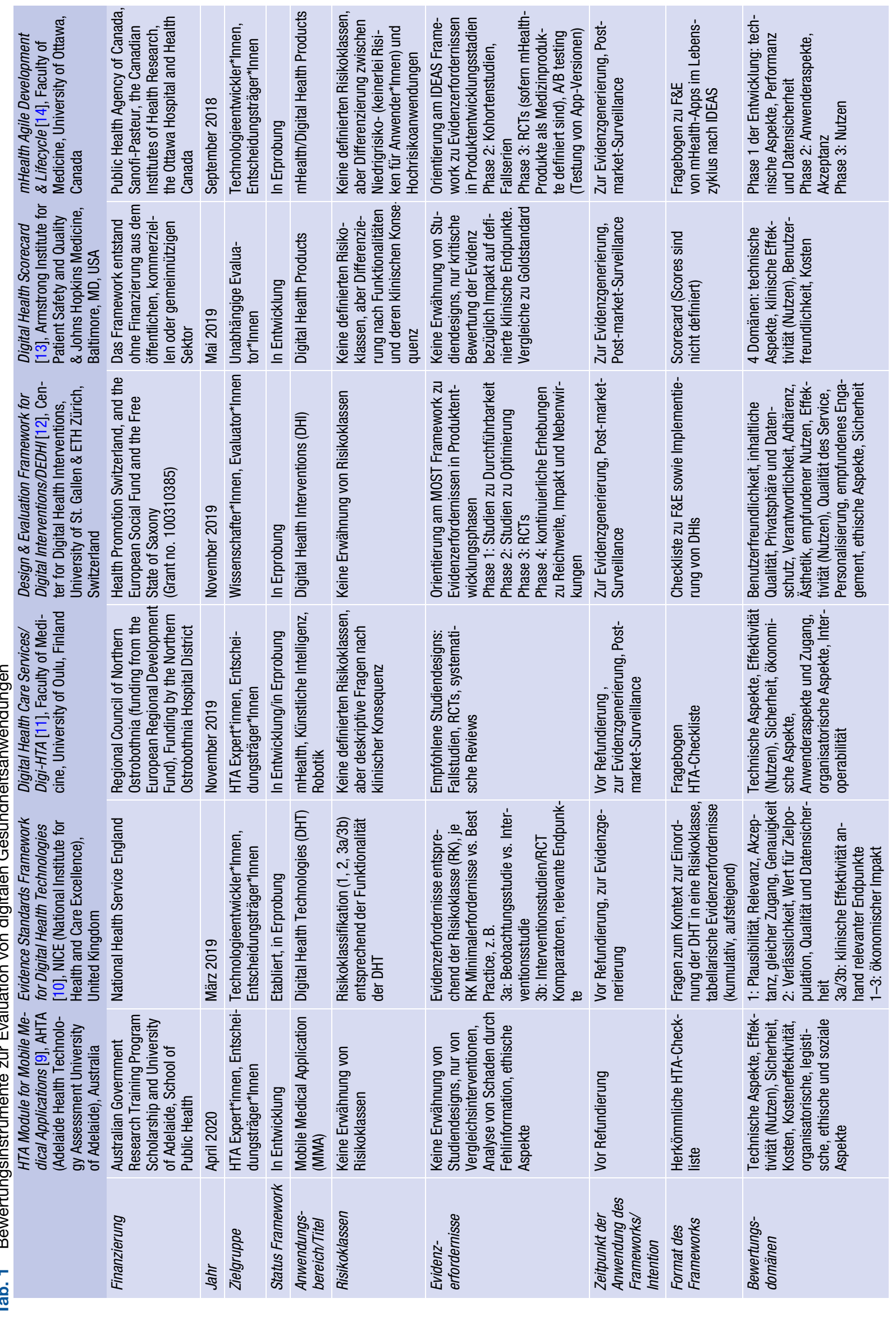




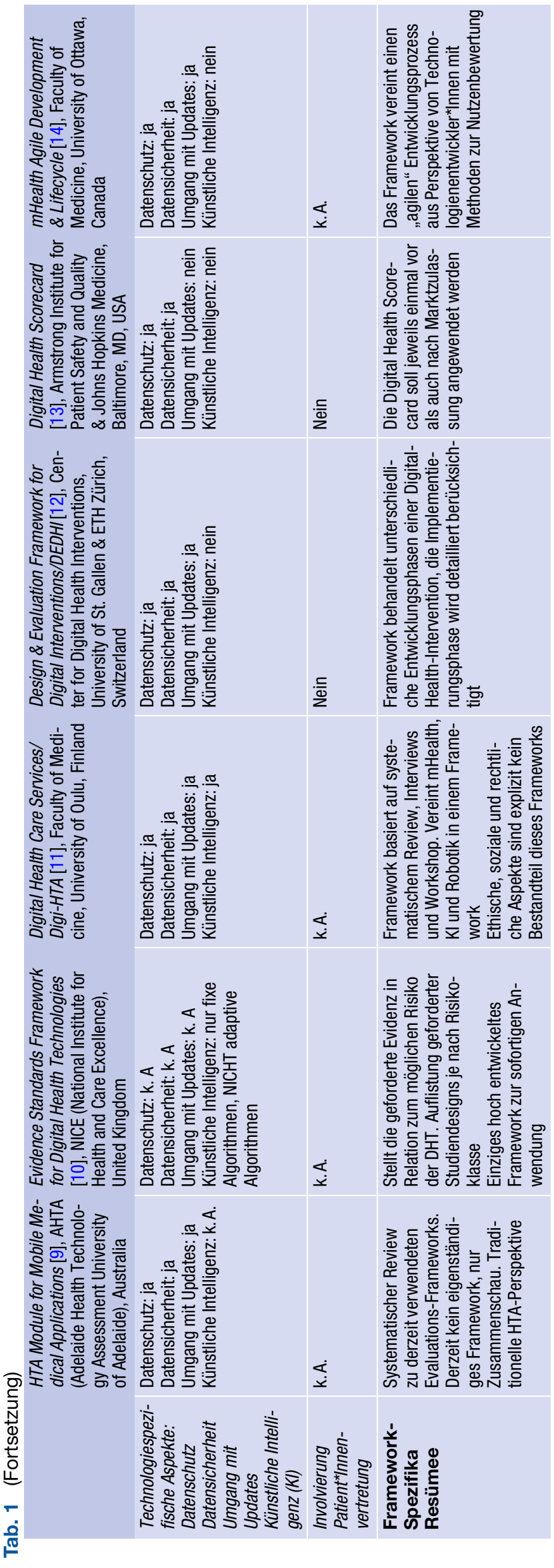

\section{Auswahl Länderstrategien und Analysekriterien:} Für die Analyse von Länderstrategien wurde eine Auswahl an Ländern mit ähnlichem Gesundheitssystem (extramurale Versorgung durch niedergelassene Ärzt*innen, deren Leistungserbringung über Verträge mit Sozialversicherungen und Krankenkassen abgegolten werden) getroffen: Deutschland, Belgien Niederlande, Frankreich. Die Auswahl der Länder basierte zusätzlich auf dem MTRC-Bericht „Reimbursement Landscape for Health Apps in Europe“ [8] und wurde durch eine Handsuche auf den Websites der entsprechenden nationalen Institutionen ergänzt. Die regulatorischen und organisatorischen Herangehensweisen der Länder wurden als Analysekriterien herangezogen.

\section{Konzeption einer Assessment-Orientierungshilfe für} Österreich: Basierend auf der Analyse und den jeweiligen Limitationen der einzelnen Bewertungsinstrumente sowie der Länderanalysen wurde zur Konzeption einer Assessment-Orientierungshilfe für Österreich ein abgestuftes Vorgehen mit Kombination von Bewertungsinstrumenten erstellt.

\section{Ergebnisse}

\section{DiGA-Bewertungsinstrumente}

Insgesamt wurden durch die systematische Literatursuche, die Befragung der INAHTA-Mitglieder sowie die in Vis et al. genannten [7] Bewertungsinstrumente für DiGA 14 Bewertungsinstrumente identifiziert [9-22]. Aus diesen wurden anhand der genannten Kriterien 6 Bewertungsinstrumente [9-14] für eine detaillierte Analyse ausgewählt, welche in Tab. 1 dargestellt sind. Die Bewertungsinstrumente wurden von Universitäten und nationalen Institutionen entwickelt.

In den Antworten der Mitglieder von INAHTA zeigte sich, dass sich viele HTA-Institutionen am National Institute for Health and Care Excellence (NICE) Evidence Standards-Bewertungsinstrument [10] (vgl. Tab. 1) orientieren und dessen Nutzung für eine erste Evaluierung von DiGA in den jeweiligen Ländern (Healthcare Improvement Scotland [HIS, Schottland], Health Technology Wales [HTW, England], Agency of Health Quality and Assessment of Catalonia [AquAS, Spanien], Canadian Agency for Drugs and Technologies in Health [CADTH, Kanada] und das Institut für Qualität und Wirtschaftlichkeit im Gesundheitswesen [IQWiG, Deutschland]) geplant ist. Ein weiteres Bewertungsinstrument wurde von Adelaide Health Technology Assessment (AHTA, Australien) entwickelt, das bisher noch keine Anwendung fand - das HTA Module for Mobile Medical Applications [9] (vgl. Tab. 1). Interesse, dieses Bewertungsinstrument zu nutzen, wurde von der Health Technology Assessment Unit (UVT, Italien) bekundet. Weitere Bewertungsinstrumente für DiGA werden derzeit von AquAS (Spanien) und dem 
Tab. 2 Risikoklassen nach 2017/745 EU-MDR und Evidenzstufen nach NICE

\begin{tabular}{|c|c|}
\hline $\begin{array}{l}\text { Risikoklassen nach EU-Verordnung 2017/745 über Medizin- } \\
\text { produkte (Regel 11) [2] }\end{array}$ & Evidenzstufen nach NICE Evidence Standards Framework [10] \\
\hline Risikoklasse 1 & Stufe 1 \\
\hline $\begin{array}{l}\text { Jede Software, die nicht in Risikoklasse 2a oder höher } \\
\text { eingeteilt wird }\end{array}$ & $\begin{array}{l}\text { Digitale Gesundheitsanwendungen ohne individuell messbare Effekte } \\
\text { auf die Gesundheit von Anwender*innen. Organisatorische Anwendungen } \\
\text { auf Systemebene } \\
\text { Beispiele: elektronische Gesundheitsakte, Krankenhaus-Informations-Systeme }\end{array}$ \\
\hline Risikoklasse 2a & Stufe 2 \\
\hline $\begin{array}{l}\text { Software, welche Information zur Entscheidung einer Dia- } \\
\text { gnose oder therapeutischen Zwecken zur Verfügung stellt, } \\
\text { ohne jedoch dabei ernstere Gesundheitsschäden verursa- } \\
\text { chen zu können (s. nachfolgende Risikoklassen) } \\
\text { Software, die physiologische Messwerte aufzeichnet, solan- } \\
\text { ge es sich dabei nicht um Vitalparameter handelt }\end{array}$ & $\begin{array}{l}\text { Digitale Gesundheitsanwendungen, welche Gesundheitsinformationen anbieten, einfache Monito- } \\
\text { ringfunktionen übernehmen, oder Anwendungen zur Kommunikation } \\
\text { Beispiele: Anwendungen mit Empfehlungen für einen gesunden Lebensstil, digitaler Kopfschmerz- } \\
\text { kalender, Anwendungen für Videosprechstunden mit Therapeut*innen }\end{array}$ \\
\hline Risikoklasse $2 b$ & Stufe $3 a$ \\
\hline $\begin{array}{l}\text { Software, welche Information zur Entscheidung einer Dia- } \\
\text { gnose oder therapeutischen Zwecken zur Verfügung stellt, } \\
\text { bei der ernstere Gesundheitsschäden oder die Notwendig- } \\
\text { keit einer chirurgischen Intervention entstehen können } \\
\text { Software, die Vitalparameter aufzeichnet } \\
\text { (Messwerte, bei denen eine Änderung eine unmittelbare } \\
\text { Gefahr für die Patient*innen darstellen kann) }\end{array}$ & $\begin{array}{l}\text { Digitale Gesundheitsanwendungen, die der Prävention und dem Selbstmanagement von Krankhei- } \\
\text { ten dienen. Digitale Gesundheitsanwendungen mit individuell messbaren Effekten auf die Gesund- } \\
\text { heit von Anwender*innen. Sie können parallel zu konventioneller Therapie eingesetzt werden } \\
\text { Beispiele: Anwendungen zur Raucherentwöhnung oder Gewichtsreduktion }\end{array}$ \\
\hline Risikoklasse 3 & Stufe $3 b$ \\
\hline $\begin{array}{l}\text { Software, welche Information zur Entscheidung einer Dia- } \\
\text { gnose oder therapeutischen Zwecken zur Verfügung stellt, } \\
\text { bei der diese Entscheidung ein Todesereignis oder irreversi- } \\
\text { ble Schäden der Gesundheit verursachen kann }\end{array}$ & $\begin{array}{l}\text { Digitale Gesundheitsanwendungen, die der Diagnostik und Therapie von Krankheiten dienen. Digita- } \\
\text { le Gesundheitsanwendungen mit klinischen Konsequenzen (durch aktives Monitoring oder Berech- } \\
\text { nungen). Digitale Gesundheitsanwendungen mit individuell messbaren Effekten auf die Gesundheit } \\
\text { von Anwender*innen } \\
\text { Beispiele: Anwendungen, die anhand eingegebener Symptome Diagnosevorschläge machen, An- } \\
\text { wendungen, die kognitive Verhaltenstherapie für Angststörungen anbieten, Anwendungen, die mit } \\
\text { einem externen Sensor oder Implantat verknüpft sind und dessen Daten automatisch und kontinu- } \\
\text { ierlich für Fernmonitoring gesendet werden }\end{array}$ \\
\hline
\end{tabular}

Institut National d'Excellence en Santé et en Services Sociaux (INESSS, Kanada) entwickelt.

Die ausgewählten Bewertungsinstrumente können für DiGA aller medizinischen Fachgebiete und Populationen eingesetzt werden (im Gegensatz zu Bewertungsinstrumenten, die sich speziell einem Fachgebiet, einer Indikation oder Population widmen). Die Bewertungsinstrumente bedienen sich unterschiedlicher Bezeichnungen („Mobile Medical Applications“, „Digital Health Technologies“, „Digital Health Interventions“ und „Digital Health Products“) und unterscheiden sich nach Format (Checkliste, Fragebogen, Tabelle), Spektrum der Bewertungsdomänen, technologiespezifischen Aspekten und Zielgruppe.

Nach Analyse der 6 ausgewählten Bewertungsinstrumente für DiGA zeigte sich, dass 4 von 6 Bewertungsinstrumenten Vorschläge zu Studiendesigns für DiGA nannten [10-12, 14]. Obwohl die Bewertungsinstrumente das Risiko bei Anwendungsfehlern mit klinischer Konsequenz zwar berücksichtigen, schlägt nur 1 Bewertungsinstrument eine präzise Einteilung nach definierten Risikoklassen der zu bewertenden DiGA vor: das NICE Evidence Standards-Bewertungsinstrument [10]. Vom NICE Evidence Standards Framework explizit ausgenommen sind jedoch DiGA, welche auf künstlicher Intelligenz mit adaptiven Algorithmen basieren (selbstlernende Algorithmen, die ihr Verhalten, basierend auf verfügbaren Informationen, kontinuierlich und automatisiert anpassen).
Wesentliche Aspekte der künstlichen Intelligenz wurden hingegen in einem von 6 Bewertungsinstrumenten berücksichtigt, dem sog. „Digi-HTA“ Framework [11]. Rechtliche, soziale und ethische Aspekte werden von diesem Bewertungsinstrument aufgrund der Komplexität und des Zeitaufwands ausgeklammert. Die Themen Datensicherheit und Datenschutz werden von diesem Bewertungsinstrument ebenfalls nicht bewertet, da deren Prüfung ausgelagert und von Datenschutzexpert*innen vorgesehen ist.

Die Evidenzstufen des NICE Evidence Standards Frameworks zeigen Ähnlichkeiten mit den Risikoklassen der EU-Verordnung 2017/745 über Medizinprodukte und sind dennoch unterschiedlich definiert (vgl. Tab. 2).

Aspekte verschiedener HTA-Domänen können je nach bewerteter DiGA als besonders relevant betrachtet werden. Für die Domäne Wirksamkeit stellt etwa diagnostische Genauigkeit eines Algorithmus oder die Reduktion von Symptomatik bei therapeutischen DiGA relevante Beurteilungskriterien dar. Für die Domäne Sicherheit gilt es, unerwünschte Nebenwirkungen wie etwa durch Fehlinformation der DiGA zu berücksichtigen. In Bezug auf Kosteneffektivität können durch DiGA Kosten vermieden, aber auch Mehrkosten verursacht werden. Als relevante organisatorische Auswirkungen sind Überdiagnostik und Interoperabilität relevante Bewertungskriterien. Die zu bewer- 
tenden Endpunkte richten sich nach Funktionsweise, Einsatzgebiet und Zweckbestimmung der DiGA.

Datenschutz bei DiGA nimmt eine besonders relevante Rolle ein, da bei Datenschutzmängeln personenbezogene Gesundheitsdaten ohne Zustimmung der Nutzer*in weitergegeben werden können. Nicht ausreichende Nachvollziehbarkeit der künstlichen Intelligenz und Intransparenz der verwendeten Algorithmen stellen ethische Problemfelder dar. Fragen zu Haftung bei Fehldiagnosen oder unerwünschten Nebenwirkungen durch DiGA stellen einen weiteren relevanten Aspekt in der Bewertung von DiGA dar.

\section{Länderstrategien}

In Europa hat man in einzelnen Ländern begonnen, regulatorische Maßnahmen zu setzen und Prozesse für eine Registrierung von DiGA aufzusetzen und infolge eine Bewertung einzelner DiGA vorzunehmen, die auch zu einer Refundierung durch Sozial- und Krankenversicherungen führen kann.

Belgien: Der administrative Rahmen für die Regulierung von DiGA bildet die Plattform mHealthBelgium (https://mhealthbelgium.be/), die auf Initiative der belgischen Regierung im Rahmen der eHealth-Strategie entstand und in enger Kooperation mit Herstellern geführt wird. Zentraler Bestandteil des Rahmenwerks ist die sog. „Validierungspyramide“ für Health-Apps. Diese Plattform zentralisiert alle relevanten und erforderlichen Informationen von CE-zertifizieren DiGA für Anwender*innen. Die Validierungspyramide besteht aus 3 Ebenen; eine App tritt immer auf der untersten Ebene (DiGA ist als Medizinprodukt zugelassen, und die Einhaltung der EU-Datenschutzverordnung wurde überprüft) ein und kann in der Hierarchie aufsteigen auf Ebene 2 (Interoperabilität und Konnektivität mit den nationalen eHealthAnforderungen wurden überprüft, Sicherheitsrisikobewertung liegt vor) und auf Ebene 3 (DiGA kann sozioökonomischen Nutzen nachweisen). Nur DiGA in der obersten Ebene werden für eine Refundierung im Rahmen der (öffentlichen) Krankenversicherungen erwogen. Bislang sind 26 Gesundheitsapplikationen auf der Plattform mHealthBelgium vertreten: 18 auf Stufe 1, 8 auf Stufe 2 und keine auf Stufe 3 der Validierungspyramide.

Frankreich: In Frankreich werden DiGA im Rahmen des bestehenden Regelwerks für Erstattungsentscheidungen zu Medizinprodukten eingegliedert. Vor der Aufnahme in die Liste der erstattungsfähigen Produkte (LPPR/,Liste des Produits et Prestations Remboursables“) werden alle Medizinprodukte von CNEDiMTS, dem Nationalen Komitee zur Evaluierung von Medizinprodukten und Gesundheitstechnologien, auf ihren Zusatznutzen evaluiert und in Klassen von diagnostisch-therapeutischem Nutzen (ASMR 1-4) klassifiziert. Die Bewertungen basieren auf 2 Methodenpapieren (2016 [23] und 2019 [24] veröffentlicht), die Nachweise auf hohem Evidenzniveau einfordern. Bislang ist eine DiGA in die Erstattungsliste aufgenommen worden.

Niederlande: Mit der Einrichtung des National eHealth Living Lab (NeLL, https://nell.eu) im Jahr 2018, einem Netzwerk aus Gesundheitsadministration, Herstellern und akademischen Institutionen, hat das niederländische Gesundheitsministerium die ersten Schritte $\mathrm{zu}$ einem einheitlichen nationalen Apps-Archiv unternommen. NeLL hat die Aufgabe, die DiGA einheitlich nach Kriterien (nachgewiesene Wirksamkeit, Anwenderfreundlichkeit und transparente Kommunikation zu Apps) zu bewerten. Derzeit sind zahlreiche Anwendungen auf der NeLL-Plattform, von denen eine die Kennzeichnung „NeLLkompatibel“ trägt.

Deutschland: In Deutschland wurden mit dem Digitale-Versorgung-Gesetz (DVG, Dez. 2019) das Verschreiben und die Refundierung von ausgewählten DiGA durch Vertragsärzt*innen und -therapeut*innen beschlossen. Voraussetzung für ein Verschreiben und eine Refundierung einer DiGA ist, dass diese im Verzeichnis erstattungsfähiger digitaler Gesundheitsanwendungen (DiGA-Verzeichnis, https://diga.bfarm. de/de) gelistet ist. Dieses DiGA-Verzeichnis wird durch das Bundesinstitut für Arzneimittel und Medizinprodukte (BfArM) verwaltet. Für DiGA mit geringem Risiko (Medizinprodukt Risikoklasse I oder IIa) wird die Bewertung durch das BfArM durchgeführt mit der Risikoklasse entsprechenden niedrigeren Evidenzanforderungen. Für DiGA mit höherem Risiko (Medizinprodukt Risikoklasse IIb oder III) wird für die Bewertung ein vollständiges HTA durch den G-BA und das IQWiG für die Evidenzbeurteilung durchgeführt. Derzeit sind 15 DiGA im Verzeichnis gelistet (Stand 18.06.2021).

Allen Herangehensweisen der beschriebenen Länder gemein ist, dass als erster Schritt die Zuständigkeit von einer verantwortlichen Institution übernommen und eine öffentlich zugängliche Plattform für verfügbare DiGA geschaffen wird. Die dort registrierten DiGA werden zunächst auf ihre CE-Zertifizierung als Medizinprodukt und auf datenschutzrechtliche Aspekte überprüft. In einem weiteren Schritt wird die Kompatibilität mit nationalen Standards der eHealthStrategien analysiert. In Ländern mit einer Wahlfreiheit unter gesetzlichen Krankenkassen (Niederlande, Deutschland) werden Versicherte auch über Zusatzangebote wie DiGA umworben. In Ländern ohne diese Wahlfreiheit (Belgien, Frankreich) ist der $\mathrm{Zu}$ gang zu einer Refundierung deutlich strikter geregelt. Große Unterschiede zeigen sich, ob Länder einen Evidenznachweis vor der Refundierungsentscheidung einfordern (Belgien) oder ob sie Evidenz generierende Refundundierungsmodelle akzeptieren oder gar mitfinanzieren (Niederlande, Deutschland). 


\section{Diskussion}

Es liegt eine Vielzahl an Bewertungsinstrumenten für digitale Gesundheitsanwendungen (DiGA) vor. Nach einer detaillierten Analyse verschiedener Bewertungsinstrumente konnte eine große Heterogenität der Bewertungsinstrumente festgestellt werden. Um bei der Vielzahl an digitalen Gesundheitsanwendungen ein effektives, zeitsparendes Assessment zu ermöglichen und andererseits die Limitationen der jeweiligen Bewertungsinstrumente zu berücksichtigen, wird als Orientierungshilfe für Entscheidungsträger*innen eine Kombination verschiedener Bewertungsinstrumente in einem abgestuften Vorgehen empfohlen.

Ein systematisches Review zu Bewertungsinstrumenten für eHealth-Anwendungen von Vis et al. [7] identifizierte und analysierte 21 Bewertungsinstrumente. Als mögliches Bewertungsmodell für eHealthAnwendungen wurde von Vis et al. eine Kombination des TEMPEST Frameworks [17], des CHEATS
Frameworks [15], des UVON Frameworks [20] und des MAST Frameworks [16] genannt. Ebenso wird das NICE Evidence Standards Framework [10] von den Autor*innen empfohlen. Der Scope des systematischen Reviews von Vis et al. [7] lag jedoch bei eHealth-Anwendungen und berücksichtigte nur teilweise die Entwicklungen im Bereich mobiler digitaler Gesundheitsanwendungen („mHealth“).

Die in dieser Arbeit analysierten Bewertungsinstrumente können für mHealth-Anwendungen eingesetzt werden. In der Analyse zeigte sich insbesondere in Bezug auf eine Einteilung in Risikoklassen sowie auf technologiespezifische Aspekte wie Datenschutz und künstliche Intelligenz eine große Heterogenität. Bisher bietet nur das NICE Evidence Standards Framework [10] eine genaue Einteilung in definierte Risikoklassen mit entsprechend geforderten Studiendesigns, es stößt bei digitalen Gesundheitsanwendungen mit adaptiven Algorithmen jedoch an Grenzen. Die Herangehensweise für die Regulierung nach Risikoklas-
Abb. 1 Empfehlung für Prozess zur Bewertung von DiGA in Österreich

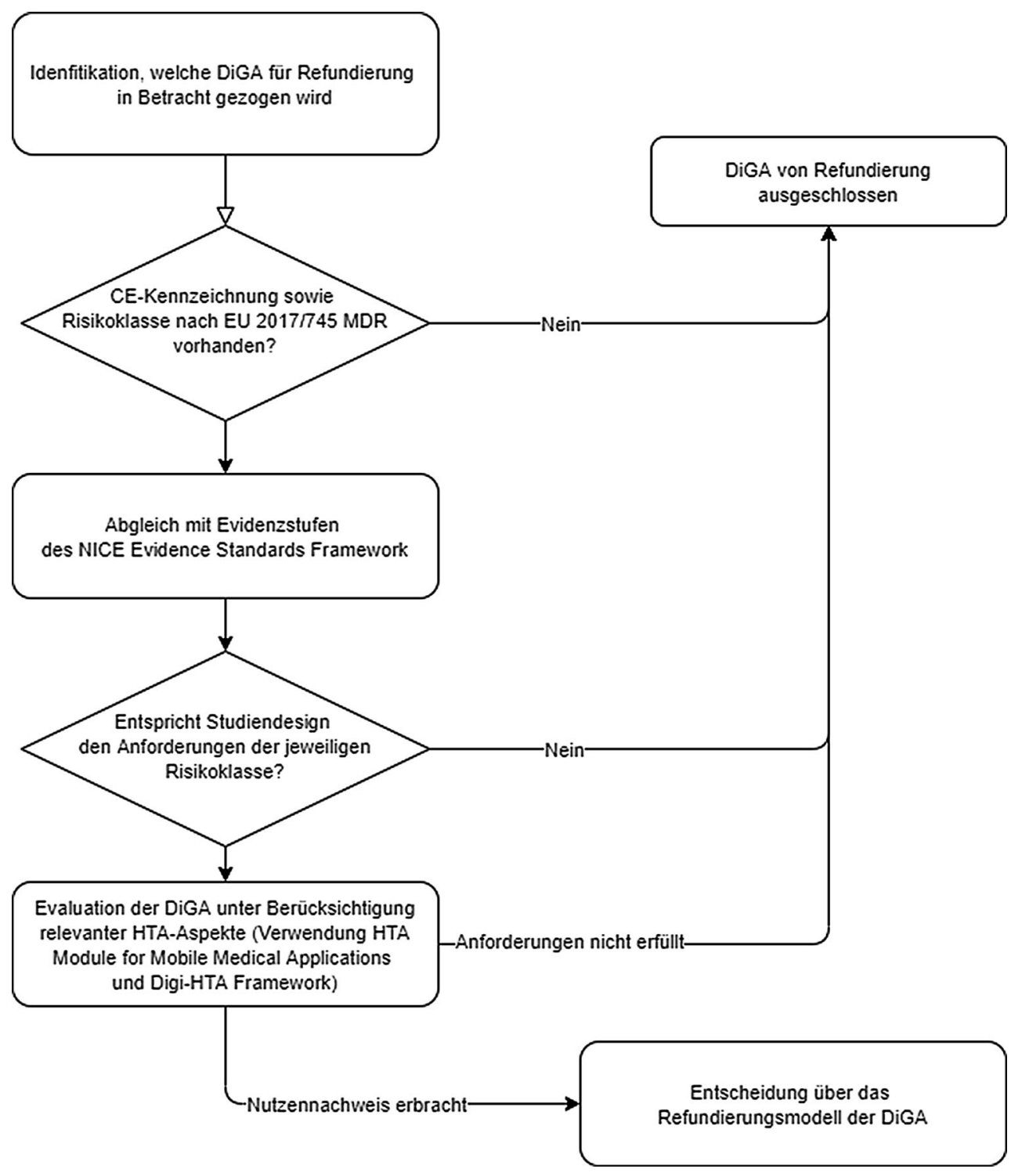


sen von DiGA wurde bereits 2016 durch das IGESInstitut empfohlen [4]. Das Digi-HTA legt einen Fokus auf Aspekte der künstlichen Intelligenz, klammert jedoch rechtliche, soziale und ethische Aspekte aus. Insgesamt bestehen für die jeweils analysierten Bewertungsinstrumente Limitationen.

Die größte internationale Aufmerksamkeit bekommt das NICE Evidence Standards Framework [10]. So empfiehlt das IQWiG in der Befragung des INAHTA die Anwendung des NICE Evidence Standards Frameworks. Zudem wurde in einer Kolumne des Netzwerks evidenzbasierter Medizin eine Visualisierung der NICE Evidence Standards FrameworkAnforderungen an neue DiGA erstellt [25]. Der hohe internationale Zuspruch für das NICE Evidence Standards Framework ergibt sich durch dessen praktische Anwendbarkeit: Mithilfe dieses Bewertungsinstruments wird eine DiGA zunächst einer funktionellen Klassifizierung nach Evidenzstufen unterzogen. DiGA, die trotz hoher Klassifizierung der Evidenzstufe 3b keinerlei Studien mit Komparator (bestenfalls randomisiert kontrollierte Studien) vorweisen können, können mithilfe dieses Bewertungsinstruments eindeutig von einer Refundierung ausgeschlossen werden.

In der Analyse ausgewählter Länder zeigt sich, dass Deutschland der gegebenen Situation (unzählige verfügbare Apps, geringe Evidenz zu Nutzenbelege) Rechnung trägt und nicht nur eine Registrierung möglicher verschreibbarer mHealth-Applikationen regelt, sondern darüber hinaus die Apps nach Risikoklassen - orientiert an der Medizinprodukteverordnung - kategorisiert und für höhere Risikoklassen (IIb und III) entsprechende Nutzenbelege durch Studien vorsieht. Diese können in Erprobungsstudien - nach Zulassung - erbracht werden. Deutschland ist mit dieser sehr klaren Vorgangsweise und der Einräumung der Möglichkeit prospektiver Evaluationsstudien (neben England) in Europa am weitesten fortgeschritten.

Eine gute Anwendbarkeit eines Bewertungsinstruments setzt eine bestmögliche Grundlage zur Entscheidungsfindung bei Refundierungsentscheidungen voraus. Auf der anderen Seite bietet ein Bewertungsinstrument auch Herstellern der Technologien eine wertvolle Orientierungshilfe für die Art von Evidenz, die für die jeweilige DiGA vorzulegen ist. Auch wenn sich DiGA von konventionellen Interventionen im Gesundheitswesen unterscheiden, sollten sie dabei dieselben Evidenzanforderungen zu Nutzen, möglichen Schäden und entstehenden Kosten erfüllen. Für evidenzbasierte Refundierungsentscheidungen einzelner digitaler Gesundheitsanwendungen bedarf es Mindestvoraussetzungen für gewünschte Studiendesigns. Nur wenige der identifizierten Bewertungsinstrumente bieten hier eine solide Orientierungshilfe. Eine Möglichkeit ist ein abgestufter Bewertungsprozess, wie er in Abb. 1 dargestellt ist.

Für Österreich wird vorgeschlagen, sich an den ersten Erfahrungen aus anderen Ländern zu orien- tieren [6]: zunächst zu überprüfen, ob eine CE-Kennzeichnung vorliegt und nach welcher Risikoklasse nach EU-Verordnung 2017/745 über Medizinprodukte [2] die DiGA klassifiziert wurde. Anschließend ist ein Abgleich der Risikoklasse nach EU-Verordnung 2017/745 mit den Evidenzerfordernissen der Evidenzstufen des NICE Evidence Standards Frameworks [10] zu empfehlen. In einem nächsten Schritt sollten ein Screening und Scoping der vorhandenen Evidenz erfolgen. Randomisierte, kontrollierte Studien, die die DiGA mit dem derzeitigen Versorgungsstandard vergleichen, stellen das höchste Maß an Evidenz dar. DiGA ohne entsprechend vorliegende Studien sollten (vorerst) von einer Refundierung ausgeschlossen werden, bis Evidenz vorgelegt wird. Für DiGA, welche die Anforderungen erfüllen, können dann die relevanten HTA-Aspekte definiert, die sich an den Erwartungen der Krankenkassen und Versprechungen der Hersteller orientieren, und infolge bewertet werden, um eine fundierte Entscheidung zum nachgewiesenen Nutzen und den erwartbaren Effekten der digitalen Gesundheitsanwendung treffen zu können. Zuletzt wird empfohlen, diesen abgestuften Prozess zu pilotieren und ggf. zu adaptieren.

Interessenkonflikt R. Jeindl und C. Wild geben an, dass kein Interessenkonflikt besteht.

Open Access Dieser Artikel wird unter der Creative Commons Namensnennung 4.0 International Lizenz veröffentlicht, welche die Nutzung, Vervielfältigung, Bearbeitung, Verbreitung und Wiedergabe in jeglichem Medium und Format erlaubt, sofern Sie den/die ursprünglichen Autor(en) und die Quelle ordnungsgemäß nennen, einen Link zur Creative Commons Lizenz beifügen und angeben, ob Änderungen vorgenommen wurden.

Die in diesem Artikel enthaltenen Bilder und sonstiges Drittmaterial unterliegen ebenfalls der genannten Creative Commons Lizenz, sofern sich aus der Abbildungslegende nichts anderes ergibt. Sofern das betreffende Material nicht unter der genannten Creative Commons Lizenz steht und die betreffende Handlung nicht nach gesetzlichen Vorschriften erlaubt ist, ist für die oben aufgeführten Weiterverwendungen des Materials die Einwilligung des jeweiligen Rechteinhabers einzuholen.

Weitere Details zur Lizenz entnehmen Sie bitte der Lizenzinformation auf http://creativecommons.org/licenses/by/4. 0/deed.de.

\section{Literatur}

1. Our World in Data. Daily hours spent with digital media, United States 2008-2018. 2020. https://ourworldindata. org/grapher/daily-hours-spent-with-digital-media-peradult-user.Zugegriffen:2. April 2021.

2. Medical Device Coordination Group. Guidance on qualification and classification of software in regulation (EU) 2017/745 - MDR and regulation (EU) 2017/746 - IVDR. 2019.

3. Gießelmann K. Medizinprodukte - Risikoklasse für Apps steigt. 2018. https://www.aerzteblatt.de/archiv/196980/ 
Medizinprodukte-Risikoklasse-fuer-Apps-steigt. Zugegriffen:2. April 2021.

4. Neumann K, Larisch K, Dietzel J, Kurepkat M, Weißer W, Wenzlau V. Digital healthcare products - leveraging opportunities - developing safe routes to market. 2016. https://www.iges.com/e6/e1621/e10211/ e13470/e15278/e15279/e15281/attr_objs15282/IGES_ Publication_Digital_healthcare_products_WEB_eng.pdf. Zugegriffen: 2. April 2021.

5. Albrecht UVH, Medizinische Hochschule Hannover. Chancen und Risiken von Gesundheits-Apps (CHARISMHA). 2016.

6. Jeindl R, Wild C. Framework zur Unterstützung von Refundierungsentscheidungen zu digitalen Gesundheitsanwendungen (mHealth) und dessen (retrospektive) Anwendung an ausgewählten Beispielen. Wien: HTAAustria; 2020.

7. Vis C, Buhrmann L, Riper H, Ossebaard HC. Health technology assessment frameworks for eHealth: a systematic review. Int J Technol Assess Health Care. 2020;36(3):204-16. https://doi.org/10.1017/S026646232000015X.

8. Med Tech Reimbursement Consulting. Reimbursement landscape for health apps in Europe. 2020.

9. Moshi MR, Tooher R, Merlin T. Development of a health technology assessment module for evaluating mobile medical applications. Int J Technol Assess Health Care. 2020;36(3):252-61. https://doi.org/10.1017/ S0266462320000288.

10. National Institute for Health and Care Excellence. Evidence standards framework for digital health technologies. 2019. https://www.nice.org.uk/Media/Default/About/whatwe-do/our-programmes/evidence-standards-framework/ digital-evidence-standards-framework.pdf. Zugegriffen: 2. April 2021.

11. Haverinen J, Keränen N, Falkenbach P, Maijala A, Kolehmainen T, Reponen J. Digi-HTA: Health technology assessment framework for digital healthcare services. Finn J eHealth eWelfare. 2019;11(4):326-41.

12. KowatschT, Otto L, HarperinkS, CottiA, SchlieterH.Adesign and evaluation framework for digital health interventions. it Inf Technol.2019;61(5-6):253-63.

13. Mathews SC, McShea MJ, Hanley CL, Ravitz A, LabriqueAB, Cohen AB. Digital health: a path to validation. NPJ Digit Med. 2019;2:38.

14. Wilson K, Bell C, Wilson L, Witteman H. Agile research to complement agile development: a proposal for an mHealth research lifecycle. NPJDigit Med.2018;1:46.
15. Shaw NT. CHEATS' : a generic information communication technology (ICT) evaluation framework. Comput Biol Med. 2002;32(3):209-20.

16. Kidholm K, Ekeland AG, Jensen LK, Rasmussen J, Pedersen CD, Bowes A, et al. A model for assessment of telemedicine applications: MAST. Int J Technol Assess Health Care. 2012;28(1):44-51.

17. Currie W. Tempest: an integrative model for health technology assessment. Health Policy Technol. 2012;1:35-49.

18. Stoyanov SR, Hides L, Kavanagh DJ, Zelenko O, Tjondronegoro D, Mani M. Mobile app rating scale: a new tool for assessing the quality of health mobile apps. JMIR mHealth uHealth.2015;3(1):e27.

19. Mummah SA, Robinson TN, King AC, Gardner CD, Sutton S. IDEAS (integrate, design, assess, and share): a framework and toolkit of strategies for the development of more effective digital interventions to change health behavior. J Med InternetRes. 2016;18(12):e317.

20. Eivazzadeh S, Anderberg P, Larsson TC, Fricker SA, Berglund J.Evaluating health information systems using ontologies. JMIR Med Inform. 2016;4(2):e20.

21. Leigh S, Ouyang J, Mimnagh C. Effective? Engaging? Secure? Applying the ORCHA-24 framework to evaluate apps for chronic insomnia disorder. Evid Based Ment Health. 2017;20(4):e20.

22. Zhou L, Bao J, Setiawan IMA, Saptono A, Parmanto B. The mHealth app usability questionnaire (MAUQ): development and validation study. JMIR mHealth uHealth. 2019;7(4):e11500.

23. Haute Authorité de Sanrté. Assessment and improvement of practice: good practice guidelines on health apps and smart devices (mobile health or mHealth). 2016.

24. Haute Authorité de Sanrté. Medical device evaluation by the CNEDiMTS (Medical Device and Health Technology Evaluation Committee): guide to the specific features of clinical evaluation of a connected medical device (CMD) in view of its application for reimbursement. 2019.

25. Sauerland S. Digitale Gesundheitsinterventionen - Wann braucht es welche Evidenz? 2019. https://www.ebmnetzwerk.de/de/medien/pdf/ebm-04_19_kvh_journal_ digitale-inventionen.pdf.Zugegriffen:7. August 2020.

Hinweis des Verlags Der Verlag bleibt in Hinblick auf geografische Zuordnungen und Gebietsbezeichnungen in veröffentlichten Karten und Institutsadressen neutral. 